Documentation et bibliothèques

DOCUMENTATION BIBLIOTHEQUES

\title{
Journal de bord d'une formation documentaire en 1998-1999
}

Library Instruction in 1998-1999: A Log Diario de a bordo de una formación documental en 1998-1999

\section{Madeleine Proulx}

Volume 45, numéro 3, juillet-septembre 1999

Formation documentaire : pistes d'action

URI : https://id.erudit.org/iderudit/1032738ar

DOI : https://doi.org/10.7202/1032738ar

Aller au sommaire du numéro

\section{Éditeur(s)}

Association pour l'avancement des sciences et des techniques de la documentation (ASTED)

\section{ISSN}

0315-2340 (imprimé)

2291-8949 (numérique)

Découvrir la revue

\section{Citer cet article}

Proulx, M. (1999). Journal de bord d'une formation documentaire en 1998-1999. Documentation et bibliothèques, 45(3), 109-115.

https://doi.org/10.7202/1032738ar

\section{Résumé de l'article}

Cet article, écrit comme un journal de bord, relate la préparation, la prestation et l'évaluation de séances de formation d'une durée de deux heures données aux étudiants inscrits en première année de baccalauréat à l'École

Polytechnique pendant l'année académique 1998-1999. Au début des sessions d'automne et d'hiver, 19 groupes totalisant 1150 étudiants ont assisté à ces formations et remis 184 travaux pratiques corrigés par le personnel de la Bibliothèque. La satisfaction de la clientèle et les résultats des travaux ont permis d'évaluer le succès des apprentissages et de modifier la participation prévue pour l'année 1999-2000.
Tous droits réservés (C) Association pour l'avancement des sciences et des techniques de la documentation (ASTED), 1999
Ce document est protégé par la loi sur le droit d'auteur. L'utilisation des services d'Érudit (y compris la reproduction) est assujettie à sa politique d'utilisation que vous pouvez consulter en ligne.

https://apropos.erudit.org/fr/usagers/politique-dutilisation/ 


\title{
Journal de bord d'une formation documentaire en 1998-1999
}

\author{
Madeleine Proulx \\ Bibliothécaire de référence et responsable de la formation documentaire \\ Bibliothèque, Service d'accès à l'information \\ École Polytechnique de Montréal
}

Cet article, écrit comme un journal de bord, relate la préparation, la prestation et l'évaluation de séances de formation d'une durée de deux heures données aux étudiants inscrits en première année de baccalauréat à l'École Polytechnique pendant l'année académique 1998-1999. Au début des sessions d'automne et d'hiver, 19 groupes totalisant 1150 étudiants ont assisté à ces formations et remis 184 travaux pratiques corrigés par le personnel de la Bibliothèque. La satisfaction de la clientèle et les résultats des travaux ont permis d'évaluer le succès des apprentissages et de modifier la participation prévue pour l'année 1999-2000.

\section{Library Instruction in 1998-1999: A Log}

This article, written in the form of a log or diary, describes the preparation, delivery and evaluation of library instruction seminars, that last two hours and were offered to first-year bachelor's degree students at the École Polytechnique during the 1998-1999 academic year. At the beginning of the autumn and winter sessions, 1150 students in 19 groups attended the sessions and completed 184 assignments that were corrected by the library staff. The satisfaction of the clients and the results of the assignments allowed the author to evaluate the success of the instruction and to modify it for the year 1999-2000.
Diario de a bordo de una formación documental en 1998-1999 Este artículo, escrito como un diario de a bordo, relata la preparación, la realización y la evaluación de las sesiones de formación de dos horas dadas a los estudiantes inscritos en primer año del priver nivel universitario de la Escuela Politécnica durante el año lectivo 1998-1999. Al comienzo de los trimestres de otoño y de invierno, 19 grupos, con un total del 1150 estudiantes, asistieron a estas sesiones de formación y presentaron 184 trabajos prácticos que fueron corregidos por el personal de la Biblioteca. La satisfacción de la clientela y los resultados de los trabajo permitieron evaluar el nivel provechoso de los aprendizajes y modificar la participación prevista para el año 1999-2000.
Au printemps 1998, une personne se présente au Bureau d'information et demande s'il est possible de préparer une formation documentaire destinée à un groupe d'étudiants de première année de baccalauréat. Ce nouveau cours de 3 crédits, intitulé Introduction au génie et aux projets d'ingénierie portant le sigle MEC1100 fera partie du programme de base que suivent tous les étudiants de première année de l'École Polytechnique de Montréal.

Les objectifs généraux de MEC 1100 visent à faire découvrir aux étudiants en quoi consiste le travail de l'ingénieur et à maîtriser les outils de communication écrite et orale par le biais d'un miniprojet d'ingénierie. En équipe de 6 , les étudiants choisissent d'améliorer un produit ou une activité de la vie de tous les jours.

L'horaire hebdomadaire du cours se décrit ainsi: (2-3-4); d'abord, 2 heures en classe consacrées aux exercices dirigés, 3 heures en classe pour les activités pédagogiques et 4 heures hors classe pour le travail d'équipe. Un professeur-coordonna- teur gère l'ensemble des activités pédagogiques. Pour l'aider dans la préparation de MEC 1100, le coordonnateur a fait appel à un étudiant au doctorat en génie mécanique qui remplit également les fonctions de chargé de cours. Cet étudiant que le Service d'accès à l'information a aidé à maintes reprises pour ses travaux est celui qui s'est présenté le 19 mai 1998 pour nous faire cette offre que nous ne saurions refuser.

\section{Objectifs et cadre pratique prévus pour la formation}

Lors de la première rencontre avec le professeur-chargé de cours, nous établissons les balises de la partie formation documentaire. II confirme que le coordonnateur désire que la Bibliothèque anime une formation documentaire d'une durée de deux périodes de 50 minutes prévue pour les exercices de la quatrième semaine de la session. Cette formation se répétera à 8 groupes de 60 étudiants en présence de leur professeur respectif pour un total de 480 étudiants.

En 1998, ni la Bibliothèque ni les laboratoires d'informatique de l'École, déjà utilisés à pleine capacité, ne peuvent accueillir autant d'étudiants pour un besoin ponctuel. Nous utiliserons donc des salles de cours d'une capacité de 65 personnes ayant un accès à Internet pour projeter sur écran les démonstrations des outils de recherche.

L'atteinte des objectifs de cette formation permettra aux étudiants de définir une stratégie de recherche, de connaître les fonctions de base du catalogue de la Bibliothèque et de découvrir quelques-uns des principaux ouvrages de référence. Les étudiants s'initieront à la recherche d'articles, de normes, de brevets, de catalogues industriels dans des banques de données bibliographiques et dans Internet. Pour évaluer et appliquer ces apprentissages, un travail pratique à faire en équipe suivra la formation et sera corrigé par le person- 
nel de la Bibliothèque.

La commande est énorme, mais elle représente une occasion unique et inespérée de rejoindre une clientèle qui brille par une faible participation (au plus 15\%) lors des formations facultatives et des visites guidées en début de session. Une clientèle qui ne craint pas d'utiliser Internet sans véritablement connaître les outils de recherche. Ce cadeau tombé du ciel réjouit le personnel de la référence ainsi que la direction de la Bibliothèque.

\section{Préparation de la formation}

De juin à la fin septembre 1998, ce projet monopolise mes activités et demande la participation du personnel du Service d'accès à l'information' 1 et des autres services. Différents types de formations déjà offertes par la Bibliothèque servent au montage du cours. Quelques idées et conseils dans des articles et des sites Internet de formations bibliographiques d'institutions universitaires d'ici et d'ailleurs s'ajoutent à nos connaissances sur le sujet².

Six rencontres avec le chargé de cours et deux rencontres avec le professeur-coordonnateur se tiennent pour faire le suivi du projet et recevoir les approbations nécessaires.

La préparation de la formation pour un grand groupe s'articule autour de trois éléments:

- Le contenu du cours,

- La préparation du travail pratique,

- La logistique des ressources humaines et matérielles.

\section{Le contenu du cours}

La formation documentaire s'inspire du déroulement d'une recherche réelle. À l'aide d'un exemple puisé dans les sujets des miniprojets de l'année précédente, nous ferons une démonstration des étapes d'une recherche documentaire. Le sujet retenu porte sur «la réduction du bruit d'une machine à laver ».

\section{Contenu de la première période de 50 minutes}

À l'entrée du local, les étudiants recevront les guides documentaires imprimés Bienvenue et Dépisteurs. Nous débute- rons par la définition du sujet et l'élaboration d'un plan de concepts. Pour expliquer cette partie, nous utiliserons des transparents et un rétroprojecteur. Nous solliciterons la participation des étudiants afin de suggérer des mots-clés. Nous apporterons des dictionnaires et encyclopédies en classe pour montrer les termes trouvés.

Nous verrons ensuite comment trouver des livres par le catalogue de la $\mathrm{Bi}$ bliothèque. Nous effectuerons des recherches titres, auteurs, mots-clés, cotes Nous expliquerons les différentes localisations de la Bibliothèque et la classification Library of Congress.

\section{Contenu de la deuxième période de $\mathbf{5 0}$ minutes}

Nous parcourrons très brièvement les sites Internet où faire la recherche de normes et de brevets, puis nous interrogerons la banque Applied science \& technology abstracts pour montrer l'application du plan de concepts dans une banque d'articles de périodiques. Nous poursuivrons par l'évaluation des documents en insistant sur les critères d'évaluation des documents trouvés dans Internet. Nous enchaînerons avec quelques recommandations concernant la rédaction d'une liste de références. Nous joindrons un petit exercice d'identification des types de documents (livre, partie d'un livre, norme, catalogue in dustriel, article de périodique) et nous terminerons par les instructions sur le travail pratique.

\section{La préparation du travail pratique}

En juin 1998, le travail pratique envisagé comportait quatre parties. La première consistait en une courte liste de cinq références pour lesquelles il fallait trouver la cote et la localisation à la Bibliothèque ainsi que le type de document parmi cinq possibilités: livre, norme, catalogue industriel, article de périodique et partie d'un livre. La deuxième touchait à l'évaluation de deux ouvrages de référence à choisir dans une liste prédéterminée. La troisième suivait avec une recherche de références reliées directement au sujet du miniprojet de chaque équipe. La quatrième et dernière touchait les citations des références bibliographiques du travail à joindre au travail final. L'ensemble du travail devait être corri- gé par le personnel de la Bibliothèque.

En août, le travail pratique prévu se modifiait selon la forme suivante: celui-ci ne comptait plus en terme de pourcentage de la note finale. Le coordonnateur avait plutôt choisi l'option de faire compter l'ensemble du travail de façon indirecte. En présentant le travail final de son miniprojet, chaque équipe devait avoir fait des recherches pour trouver des références et devait les citer correctement. Donc, les parties 3 et 4 énoncées dans le paragraphe précédent se fondaient dans le travail final qui serait corrigé en totalité par les professeurs. Pour les parties 1 et 2 , nous les conservions sous leurs formes initiales et chaque professeur en exigerait la réalisation en le qualifiant d'essentiel pour la réussite du miniprojet. Le personnel de la $\mathrm{Bi}$ bliothèque s'assurait de corriger les parties 1 et 2 sans que cette notation figure dans le résultat final.

Malgré notre déception et un certain scepticisme au regard de ce contrôle des apprentissages, notre participation s'est poursuivie avec beaucoup d'optimisme face à cette occasion de rejoindre autant d'étudiants à l'intérieur d'un cours inscrit au programme obligatoire. Afin d'éviter le plagiat et de diversifier les ressources, nous avons conçu dix listes différentes de références à identifier: la Figure 1 en est un exemple. La Figure 2 mentionne les questions d'évaluation de documents de référence. La question $2 a$ donne le choix entre trois encyclopédies tandis que la question $2 b$ laisse à l'étudiant la liberté d'évaluer n'importe quel ouvrage mentionné dans un des guides documentaires de la série Les Dépisteurs. Les Dépisteurs étant des listes d'ouvrages choisis de la Bibliothèque dans un domaine du génie.

\section{La logistique des ressources humaines et matérielles}

En mai 1998 , le projet initial prévoyait 8 groupes d'environ 60 étudiants. En juillet, le professeur m'informe qu'il y aura 2 groupes de plus et que les séances auront lieu en même temps, ce qui signifie l'implication d'un autre bibliothécaire pour la prestation du service. Nous sommes

\footnotetext{
1. Le Service d'accès à l'information regroupe les services de référence et de fournitures de documents.

2. Voir les sources consultées à la fin de l'article.
} 
Figure 1. Travail pratique - Sessions d'automne et d'hiver 1998-1999 Partie 1. Identification de références

\begin{tabular}{|c|c|c|c|c|}
\hline \multicolumn{5}{|c|}{ Partie 1(15 points) } \\
\hline & $\begin{array}{l}\text { chacune des cinq références suivantes : } \\
\text { Identifiez le type de documents. (S'agit-il d'un livre, d'un article de pério- } \\
\text { dique, d'un catalogue industriel, d'une partie d'un livre ou d'une } \\
\text { norme?) } \\
\text { Dans le catalogue de la Bibliothèque, trouvez la cote et la section de } \\
\text { chaque document. }\end{array}$ & TYPE & SECTION & COTE \\
\hline 1 & $\begin{array}{l}\text { HUBBARD, Mont. "Simulating sensitive dynamic control of a bobsled". } \\
\text { Simulation. Vol. } 65 \text {, Aug. 1995. p. 147-51. }\end{array}$ & $\begin{array}{l}\text { Article de } \\
\text { periodique }\end{array}$ & Revues & $\begin{array}{l}\text { TA } \\
343 \\
S 52\end{array}$ \\
\hline 2 & $\begin{array}{l}\text { PHILIBERT, Jean. Métallurgie: du minerai au matériau. Paris: } \\
\text { Masson, c1998, } 1107 \mathrm{p} .\end{array}$ & Livie & GEN & $\begin{array}{l}\text { TN } \\
665 \\
M 48 \\
1998\end{array}$ \\
\hline 3 & $\begin{array}{l}\text { CLAROSTAT MFG. CO. Photoelectric products catalog. S.L.: the CO, } \\
\text { c1993. } 130 \text { p. }\end{array}$ & $\begin{array}{l}\text { Catalogue } \\
\text { Industriel }\end{array}$ & CAT-1 & $\begin{array}{l}\mathrm{TJ} \\
223 \\
\mathrm{~T} 5 \\
5201\end{array}$ \\
\hline 4 & $\begin{array}{l}\text { ASSOCIATION CANADIENNE DE NORMALISATION. Huiles isolantes } \\
\text { électriques pour transformateurs et matériels de coupure. Etobicoke, } \\
\text { Ont. : I'Association, 1997. } 7 \text { p. Norme nationale du Canada } \\
\text { CAN/CSA-C50-97. }\end{array}$ & Norme & NORMES & $\begin{array}{l}\mathrm{CA1} \\
\mathrm{CC} .3 \\
50(\mathrm{~F})\end{array}$ \\
\hline 5 & $\begin{array}{l}\text { McMAHON, C. J. "Bicycle materials". The Encyclopedia of advanced } \\
\text { materials. Oxford, England : Pergamon, c1994.Vol.1, p. 232-238. }\end{array}$ & $\begin{array}{l}\text { Partie diun } \\
\text { Inve }\end{array}$ & DICT & $\begin{array}{l}\text { TA } \\
404.8 \\
\text { E63 } \\
1994 \\
\text { v. } 1\end{array}$ \\
\hline
\end{tabular}

Figure 2. Travail pratique - Session d'automne 1998

Partie 2. Évaluation d'ouvrages de référence

Partie 2 (20 points)

a) En vous basant sur le sujet de votre miniprojet, évaluez un des documents suivants en indiquant deux points forts et deux points faibles par document Indiquez où dans le document vous avez trouvé des informations (pages, sections, paragraphes...)
Techniques
de l'ingénieur
ou McGraw-Hill science and
technology encyclopedia
Dict TA 9 T4C
Dict Q 121 M3 1997
ou Encyclopedia of
physical science and
technology
Dict QC 5 E63 1992

Sujet du miniprojet:

Pages, sections consultées:

b) En vous basant sur le sujet de votre miniprojet, évaluez un ouvrage de référence de votre choix parmi ceux mentionnés dans la section Aide mémoire (Handbooks) d'un Dépisteur. Donnez le titre, la section et la cote du document ainsi que les pages consultées.

donc rendus à une formation qui touchera 600 étudiants. Finalement, en août, le nombre de groupes est porté à 12 pour une moyenne de 65 étudiants. À l'automne 1998, nous rejoindrons 775 étudiants par le biais de ce cours.

Dès la fin juin, nous aurons le souci de préparer les ressources pour desservir un nombre croissant d'utilisateurs. De nouveaux dictionnaires, index, ouvrages de référence, documents mis à la réserve s'ajoutent pour répondre à la demande.

Trois cents sites Internet choisis deviendront les Meilleurs sites Internet pour étudiants en génie (http://www.biblio.polymtl.ca/meilleurs/meilintro.html) afin de faciliter la recherche et le repérage de documents de valeur dans Internet. Cette liste, dont les groupes de MEC1100 seront les premiers bénéficiaires, fait partie des outils mis à leur disposition par la Bibliothèque pour planifier et exécuter leurs recherches.

En août, avant le début de la session, des problèmes de nature technique se manifestent: absence d'accès au réseau informatique et de logiciels disponibles sur les ordinateurs. Ces problèmes nécessitent l'intervention du Service informatique. Malgré des demandes clairement définies, ce n'est que la veille du début des formations que nous sommes invités à nous déplacer dans les locaux pour tester les liens au réseau, les codes d'accès personnalisés hors du site de la Bibliothèque et à nous familiariser avec les canons projecteurs.

Tout au long de ces préparatifs, la responsable du Service d'accès à l'information facilitera les contacts que j'aurai à établir avec toutes les autres personnes de l'École, notamment le personnel du Service informatique et du Bureau d'appui pédagogique. Mon collègue, bibliothécaire de référence, agira comme formateur pour 5 groupes. Une technicienne de la référence veillera à ce que suffisamment de guides documentaires soient imprimés et livrés pour la rentrée. Pendant la semaine de formation, le bibliothécaire responsable du développement des collections prêtera main forte aux techniciennes à la référence. Je peux donc compter sur un soutien important de mes collègues.

\section{Session automne 1998}

\section{Semaine de formation - 18 au 24 septembre 1998}

18 septembre, 10 heures $30,1^{\mathrm{er}}$ groupe. Le coordonnateur du cours, professeur de ce premier groupe, me présente et c'est sous un délire d'applaudissements que je suis accueillie. Oups! Disons que ce n'est pas tout à fait comme ça que cela s'est passé. En réalité, le professeur ne savait pas trop à quoi s'attendre puisqu'il n'avait jamais assisté à une séance de formation depuis très longtemps.

Je débute donc et malgré le fait que tout se déroule sans problème, j'ai peine à 
compléter le plan de cours en deux périodes. Je m'explique: donner un cours dans un local de formation dont l'équipement vous est familier pour un groupe de 15 personnes et se retrouver avec un groupe d'environ 65 étudiants, qui vont et viennent, font du bruit avec leur chaise, renversent un café ou discutent entre eux, dans un local où les équipements viennent d'être mis en place ne représente pas la même dynamique.

À la fin des deux périodes, je ne dispose que d'une minute pour présenter le travail qui reçoit un accueil vraiment glacial. Cette réception m'étonne un peu puisqu'il est bien écrit dans le cahier des étudiants que ce devoir est prévu. Mais je vois bien que plusieurs sont sous le choc comme s'ils n'avaient pas été prévenus. Même le professeur hésite quelque peu à répondre à ceux qui demandent: "Ça compte pour combien ce travail-là?» Cette ambiguïté autour de la notation non comptabilisée du travail pratique se manifestera chez tous les groupes et s'exprimera négativement à maintes reprises.

Durant les formations, les étudiants ont semblé attentifs surtout pendant la première période. Plusieurs posent des questions, sur la collection, sur le catalogue, sur les compressions budgétaires, sur les banques de données accessibles par Internet. Parmi les 12 groupes, certains sont plus intéressés et d'autres plus passifs.

Les professeurs et les chargés de cours manifestent dans l'ensemble une grande satisfaction de notre participation.

\section{Semaine suivant la formation - 25 septembre au 1er octobre 1998}

Durant la semaine suivant la formation, notre rôle consistait à aider les étudiants à compléter leur travail et à recevoir leurs travaux. À cet égard, tout le personnel du Service d'accès à l'information, soit 3 bibliothécaires à plein temps incluant la responsable, 3 techniciennes en documentation dont 2 à mi-temps et 3 auxiliaires participent activement à répondre à ces demandes accrues d'assistance. Une hausse moyenne de $63 \%$ de documents de référence et de dictionnaires à ranger pour les mois de septembre, octobre et novembre confirmera notre impression de recherches intensives menées par les étudiants. Un matin, le personnel comparera la section des encyclopédies à un magasin lors d'une «vente de feu » tellement l'affluence y était importante.

Les questions qui nous sont posées se rapportent surtout à l'évaluation des ouvrages de référence et font état de difficultés à comprendre ce qui est demandé. Les étudiants s'attendent à ce que les informations contenues dans les documents que nous leur demandons d'évaluer correspondent exactement au sujet de leur miniprojet. Peu d'entre eux savent comment consulter les encyclopédies. Les étudiants utilisent sans distinction les différentes éditions des documents proposés.

\section{Semaines de correction - 5 au 16 octobre. 1998}

Nous corrigerons 129 travaux pratiques en deux semaines. La partie 1 (Identification, localisation et cote des références) sera notée par les techniciennes du service selon un barème préétabli tandis que la partie 2 (Évaluation des ouvrages) sera corrigée par les bibliothécaires (Figures 1 et 2 ).

Les résultats noùs donnent des indications assez révélatrices. Les résultats sont assez élevés pour la partie 1 avec une moyenne de $88 \%$. L'identification la moins réussie est celle d'une partie de livre ou partie de document tandis que toutes les autres catégories (livre, norme, catalogue industriel, article de bibliothèque) ne posent pas de difficulté.

Pour la partie 2, c'est un demi-succès puisque les moyennes de $73 \%$ et de $75 \%$ pour les deux sous-questions $a$ et $b$ reflètent davantage notre surprise à la vue des réponses que la qualité ou la véracité du contenu que nous souhaitions à ces réponses. La réaction de surprise passée, nous avons réalisé que les étudiants auraient eu besoin d'explications plus détaillées sur cette partie du travail. Nous avons donc été généreux dans la correction en se promettant de corriger ce manque de précision dès la session d'hiver 1999.

À la partie 2a (voir Figure 2), les étudiants ont surtout répondu s'ils trouvaient ou non de la documentation en relation directe avec leur sujet de miniprojet. Le document était bon si l'étudiant y avait trouvé de l'information sur son sujet, sinon il n'était pas bon. Par exemple, si le sujet du miniprojet était d'améliorer une pelle à neige pour réduire l'effort du ramassage, les étudiants cherchaient «pelle» dans l'encyclopédie Techniques de l'ingénieur. Les résultats étaient nuls bien sûr. II ne leur venait pas à l'esprit de chercher avec' des termes tels «ergonomie » ou « conception d'outil mécanique».

Les autres commentaires les plus fréquents concernaient l'aspect «cosmétique " du document, les illustrations et schémas étaient clairs ou trop complexes, il n'y en avait pas en couleur, le document était en anglais, l'information n'était pas à jour. Nous avons aussi constaté que les étudiants se trompaient d'édition et indiquait que l'information contenue était périmée, alors qu'ils avaient consulté une édition de 1980 plutôt que celle recommandée de 1997.

À la partie $2 \mathrm{~b}$, les réponses ont été similaires en ce sens; encore une fois, un bon document contenait de l'information sur le sujet de leur miniprojet tandis qu'un mauvais document n'en contenait peu ou pas. Les difficultés les plus courantes se répartissaient ainsi: réponse partielle, réponse hors contexte, document absent de la liste des Dépisteurs, mauvaise façon de chercher dans un ouvrage de référence; d'autres ont évalué des documents sans nous indiquer ceux qu'ils avaient choisis.

\section{Autoévaluation du cours - 2 au 6 novembre 1998}

Deux semaines après la remise des corrections, nous sommes retournés dans 3 groupes sur 12 pour demander aux étudiants de remplir un formulaire d'appréciation de la formation. Nous avons donc récolté 195 répondants sur 775 .

Les principales réactions se répartissaient ainsi: Très satisfait: $53 \%$, Satisfait: $34 \%$, Insatisfait: $12 \%$, Très insatisfait: $1 \%$.

Les commentaires les plus fréquents se lisaient comme suit: trop long, trop court, difficile de tout se rappeler, très utile pour leur recherche, inutile car ils connaissent déjà tout ça.

Les suggestions ciblaient la production d'un document imprimé reproduisant, selon l'ordre de présentation, les étapes d'une recherche efficace ainsi que l'amélioration de la fiabilité des équipements informatiques.

Les 12 professeurs ont pour la plupart commenté la formation le jour même où elle fut donnée et 8 d'entre eux ont été très satisfaits de notre participation en souhai- 
Figure 3. Semaine suivant la formation - 25 septembre au $1^{\text {er }}$ octobre 1998 Partie 2. Plan de concepts et Évaluation d'ouvrages de référence

\section{Partie 2 (20 points)}

a) En vous basant sur le sujet de votre miniprojet, rédigez votre plan de concepts comprenant

$>$ votre sujet,

- l'identification des concepts (maximum 3 concepts),

- les mots-clés anglais et français de chaque concept

> la stratégie de recherche rédigée en une phrase en utilisant les opérateurs booléens OU, ET, l'opérateur de troncature * et les parenth ses (). Utilisez une feuille supplémentaire si vous manquez d'espace.

Sujet

Concept $n^{\circ} 1$

Concept $n^{\circ} 2$

Mots-clés concept $n^{\circ} 1$ :

Mots-clés concept n²:

Stratégie de recherche:

b) En vous basant sur les critères d'évaluation des documents mentionnés durant le cours, évaluez un des 6 documents suivants en indiquant deux points forts et deux points faibles du document. Servez-vous du sujet de miniprojet pour faire cette évaluation. Indiquez les pages ou sections consultées ainsi que les mots utilisés pour faire votre recherche.

Techniques de l'ingénieur Mcgraw-Hill science and technology Encyclopedia of physical science and

Dict TA 9

http://www.techniques-ingenieur.fr/ $\square$

Encyclopedia of applied physics encyclopedia Encyclopedia technology

Dict Q 121 M3 $1997 \square$ Dict QC 5 E63 1992

Encyclopedia of applied physics
Dict QC 5 E62 $1991 \square$ Encyclopedia of advanced materials Dict TA 404.8 E63 $1994 \square$

Encyclopedia of computer science Dict QA 76.15 E48 $1995 \square$

Pages ou sections consultées:

Mots utilisés pour consulter l'ouvrage:

Point fort $n^{\circ} 1$

Point fort $n^{\circ} 2$

Point faible $n^{\circ} 1$

Point faible $n^{\circ} 2$

tant que nous donnions cette formation plus tôt à la session prochaine.

Concurremment à notre démarche, le Bureau d'appui pédagogique, par le biais d'un formulaire, a procédé à l'évaluation de l'ensemble du cours MEC1100, y compris la formation documentaire. Nous avons reçu les résultats après les formations de janvier-février 1999 et nous y reviendrons plus loin au point «Appréciation de la formation documentaire en relation avec le cours MEC1100».

\section{Bilan décembre 1998}

À la fin de la session, mes collègues et moi avons tenu une réunion pour faire le point sur la formation, évaluer les résultats et améliorer le produit.

Nous avons alors convenu de limiter la partie normes et brevets pour donner plus de place à la préparation d'un plan de concepts et avoir plus de temps pour expliquer le travail pratique.
À propos du travail pratique, nous avons modifié la partie 2 pour demander en question $2 a$ de produire le plan de concepts de leur miniprojet et en $2 b$ d'évaluer un document choisi parmi 6 encyclopédies en s'inspirant des critères de qualité présentés en classe. La Figure 3 reproduit les questions modifiées de la session hiver 1999.

Finalement, un feuillet guide de 4 pages s'inspirant de la chronologie de la formation s'est aussi ajouté en vue de répondre aux suggestions recueillies lors de l'évaluation.

\section{Session Hiver 1999}

\section{Semaine de formation - 29 janvier au 3 février 1999}

Après les vacances de Noël, nous recommençons le processus pour 376 étu- diants répartis en 7 groupes. Le coordonnateur préférant attendre la fin de l'année académique pour modifier l'ordre des activités, nous conservons la quatrième semaine de la session pour la formation documentaire. Les seuls changements seront ceux que nous apporterons suite au bilan de décembre 1998 .

Nous observons sensiblement les mêmes réactions des étudiants: attention plus soutenue pendant la première période, questions variées sur l'accès à distance du catalogue, les particularités d'interrogation, les compressions budgétaires. La réaction des professeurs ne change pas.

Cette reprise de formation un peu plus allégée par son contenu et plus précise dans les attentes du travail à faire nous permet de mieux respirer pendant cette semaine et d'observer davantage notre clientèle. Nous nous permettrons même d'inviter des étudiants à venir nous remplacer à l'avant pour taper au clavier les commandes et recherches illustrant les exemples ou encore de comparer des stratégies de recherche pour un même sujet avec des outils différents.

\section{Semaine suivant la formation - 5 au 12 février 1999}

Cette période génère moins d'effervescence qu'à la session d'automne. Peut-être verrons-nous une meilleure compréhension des questions et un succès plus directement lié à des directives claires sur le travail à faire. Nous recevrons 55 travaux pratiques, soit en proportion avec le nombre d'étudiants, une baisse de $15 \%$ par rapport à la session d'automne. Nous nous y attendions puisque le bouche à oreille entre étudiants propage l'information que "Ça ne compte pas», et ce, malgré un appui des 7 professeurs à promouvoir le travail comme étant essentiel à la suite des recherches et du rapport final qui compte pour $25 \%$ de la note globale.

\section{Semaines de correction - 15 au 26 février 1999}

Les résultats des questions d'identification, partie 1 (Figure 1), ont été similaires aux résultats de la session d'automne, soit une moyenne de $87 \%$. 
La refonte de la partie 2 (Figure 3), la question $2 a$ leur demandant d'écrire leur sujet, d'identifier les concepts et leurs mots-clés et d'écrire leur équation de recherche - nous amène un surcroît de travail, mais également des informations très précieuses sur la compréhension réelle des étudiants de notre enseignement. En effet, les étudiants obtiennent une moyenne de $55 \%$ après une correction généreuse compte tenu des efforts fournis. Nous avons pu constater jusqu'à quel point cette étape d'apparence facile représentait un défi de taille pour que ces étudiants puissent mener avec succès des recherches avec des outils de base comme le catalogue de la Bibliothèque et la banque Applied science and technology abstracts. Nous avons aussi compris que pour répondre à leurs besoins de documentation en relation avec leur miniprojet, les meilleurs outils étaient les banques de brevets et la banque ASTA. Or, nous avions presque éliminé les brevets dans notre formation. De plus, le fait qu'ils ne comprennent pas l'importance du vocabulaire dans leur recherche nous obligeait à remettre en question l'ensemble de la formation.

L'évaluation de l'ouvrage de référence, question $2 b$, s'est détériorée malgré plus d'explications des critères d'évaluation et un choix plus ciblé des ouvrages. À cette question, la moyenne atteinte chutera à $48 \%$. Nous expliquons ce résultat de deux façons. D'abord, nous avions été généreux en première session en croyant que nos explications manquaient de clarté. Notre correction d'hiver était plus rigoureuse. En plus, les étudiants de première année manquent d'expérience et de connaissances dans leur domaine pour déduire, à partir d'un cas concret, les notions théoriques que contiennent les applications d'ingénierie. Ils ne réalisent pas encore que l'amélioration de la pelle à neige nécessite l'acquisition de connaissances en biomécanique, en anthropométrie et en ergonomie.

Ce qui ne signifie pas nécessairement que l'exercice est inutile ou qu'il est un échec. Cependant, nous avons compris qu'il fallait revoir notre enseignement pour permettre aux étudiants d'acquérir cette maturité de pensée et procéder par étapes.

Nous avons donc transmis les résultats des corrections incluant des suggestions de réorientation de la recherche, des notices d'articles de périodiques et des échantillons de brevets pour démontrer aux étudiants ce qu'il était possible de trouver avec une stratégie bien organisée. Nous avons laissé retomber la poussière jusqu'au jour où le conseiller pédagogique associé à ce cours nous a communiqué les résultats de l'évaluation de l'activité bibliothèque dans l'ensemble du cours.

\section{Évaluation de la formation documentaire pour l'année 1998-1999}

\section{Appréciation de la formation documentaire en relation avec le cours MEC1100}

Selon les résultats obtenus par le Bureau d'appui pédagogique, des neuf exercices pratiques de l'ensemble du cours MEC1100 de la session automne 1998, la formation documentaire occupait l'avant dernière position avec un taux de satisfaction de $53 \%$ tandis que notre autoévaluation indiquait un taux de satisfaction de $87 \%$.

L'activité la plus appréciée, Travail de l'ingénieur, récoltait $73 \%$ et la moins appréciée, L'ingénieur et l'ordinateur, se situait à $48 \%$. Il faut cependant préciser que 2 des 5 critères d'évaluation s'appliquaient peu à notre participation.

Les critères du Bureau d'appui pédagogique étaient les suivants:

1. les exercices ont bien réussi à me faire découvrir en quoi consiste le métier d'ingénieur;

2. les exercices m'ont confirmé que le génie me convenait comme choix de carrière;

3. le déroulement des exercices convenait bien pour échanger et discuter des informations recueillies;

4. les directives des exercices étaient claires;

5. la quantité de travail hors classe exigée pour les exercices était correcte.

\section{Bilan avril 1999}

Compte tenu des critères de l'autoévaluation de novembre 1998, des résultats des travaux pratiques, des commentaires du personnel de la référence et de l'ensemble de notre expérience, nous avons mis en évidence les points suivants:
- Les brevets et articles de périodiques sont les sources documentaires qui répondent le plus aux objectifs à atteindre par les étudiants inscrits à ce cours.

- La formation, même appréciée, contient trop de notions à assimiler en une seule fois.

Nous ne disposons toujours pas de laboratoires informatiques pour que les étudiants appliquent concrètement la démarche proposée, reçoivent des conseils et améliorent avec succès leur recherche.

Un travail pratique noté qui ne compte pas prête à confusion sur le rôle de la bibliothèque et fait obstacle à la collaboration que nous voulons établir avec notre clientèle.

- La méthode adoptée pour donner notre exposé (cours magistral) offre peu d'interactions avec les étudiants.

Devant ce constat, nous avons retravaillé le contenu et avons demandé au conseiller pédagogique d'examiner de nouveaux scénarios de formation afin de profiter de son expertise dans le domaine. Nous avons ainsi conçu une nouvelle façon d'aborder notre activité de formation pour l'année 1999-2000. Nous avons réduit le nombre d'objectifs, nous avons créé des activités où la recherche s'effectuerait concrètement par les étudiants. Nous avons ciblé la réalisation d'un plan de concepts, la recherche de brevets, la recherche de catalogues industriels et la recherche dans le catalogue de la Bibliothèque.

\section{Rencontre avec l'équipe de professeurs}

Le coordonnateur du cours et le conseiller pédagogique nous ont invités à participer à la réunion bilan de la session hiver 1999 pour le cours MEC1100 avec tous les professeurs et chargés de cours impliqués.

Le fait de participer à une réunion du groupe de professeurs et d'obtenir aide et appui du conseiller pédagogique nous a donné un poids et un moyen de rencontrer les intervenants de première ligne dans la formation des étudiants.

Durant les dix minutes consacrées à l'activité de formation documentaire, nous avons recueilli des commentaires confirmant notre propre bilan et avons présenté différentes propositions pour l'année 1999-2000. 


\section{Conclusion}

Notre participation de l'année 19981999 , en fonction des demandes des professeurs, que ce soit pour les 19 séances de formation ou pour le support du personnel de la référence aux recherches des étudiants, nous a permis de rejoindre 1150 nouveaux étudiants et représente un succès en soi.

Les portes de la collaboration avec l'équipe de professeurs de MEC1100 nous sont maintenant ouvertes.

Notre implication dans la correction de 184 travaux pratiques, incluant 55 plans de concepts ainsi que la cueillette de commentaires et suggestions des étudiants, professeurs et conseiller pédagogique, nous ont donné une appréciation réaliste de l'atteinte de nos objectifs.

Les étudiants ont appris à bien utiliser le catalogue de la Bibliothèque, à découvrir des ouvrages de référence, mais éprouvent des difficultés importantes à établir correctement un plan de concepts.

L'expérience de l'année 1998-1999 mène à des propositions d'activités de formation pour l'année 1999-2000 qui ont été bien accueillies par les professeurs, le coordonnateur et le conseiller pédagogique.

En août 1999, en semaine 2, nous animerons une activité de deux périodes de 50 minutes pour la réalisation de plans de concepts. Aucun travail supplémentaire ne sera requis par la Bibliothèque. Nous offrirons aux équipes d'étudiants une consultation avec un bibliothécaire durant la semaine 3 pour compléter leur plan et démarrer la recherche avec les outils adéquats.

En septembre 1999, en semaine 4, deux périodes de 50 minutes s'ajouteront pour la présentation des Meilleurs sites Internet pour étudiants en génie http:// www.biblio.polymtl.ca/meilleurs/intro.html et pour une plénière sur les résultats de 3 recherches différentes effectuées par des étudiants touchant les brevets, les catalogues industriels et le catalogue de la $\mathrm{Bi}-$ bliothèque.

Ces nouvelles formations atteindrontelles leurs objectifs d'améliorer les habiletés de recherche des étudiants? Je le souhaite vivement et, qui sait, peut-être y aura-t-il une suite à cet article l'an prochain. À suivre....

\section{Sources consultées}

Bartels, Carrie and Joni Kanzler. (Page consultée le 25 février 1998). Innovative ways to teach library instruction in two different academic environments. [En ligne] Adresse URL: http:// www.bsu.edu/library/internal/ala/poster.htm

Brewer, Janet. ( Page consultée le 25 février 1998). Show me the process. [En ligne]. Adresse URL: http ://www.mursuky.edu/msml/poster/

Brown, Marthe et al. (Page consultée le 5 mars 1998). Un message des bibliothécaires. [En ligne]. Adresse URL: http://www.laurentian.ca/www/library/ateliers.html

Campeau, N. et O. Paradis. 1997. La maîtrise de l'information et la formation des ingénieurs à l'École Polytechnique de Montréal. $8 \mathrm{p}$.

La Cité collégiale. (Page consultée le 5 mars 1998). Introduction à la recherche documentaire. [En ligne]. Adresse URL: http://www.lacitec.on.ca/ webdoc.formatio.htm

Engle, Michael. (Page consultée le 25 février 1998). The seven steps of the research process.[En ligne] Adresse URL: http://www.library.cornel.edu/okuref/research/skill1.htm

Grondin, Luc. (Page consultée le 25 février 1998). Formation documentaire. [En ligne]. Adresse URL: http://www.unites.uqam.ca/bib/thematique/ sciences/W3/formations.html

Holmes, Colette O., Elizabeth D. Irish and Thomas C. Haley. 1994. BI for an undergraduate engineering course: an interactive model for a large-enrollment course. Research strategies 12 (Spring): 115-121.

Laverty, Cory. (Page consultée le 14 septembre 1998). Resarch strategy: Queen's University libraries.[En ligne]. Adresse URL: http://stauffer.queensu.ca/inforef/strategy.htm

Roddy, Kevin M. (Page consultée le 25 février 1998). How to build librarian /Instructional faculty collaborative partnerships. [En ligne]. Adresse URL: http ://www2.hawaii.edu/ kroddy/poster/

Thomes, Kate, Evan Cornell and Byron Gottfried. (Page consultée le 25 février 1998). Teaching freshmen to write technical reports and to navigate the library: a win-win situation. In 1997 ASEE/IEEE frontiers in education conference. [En ligne] Adresse URL: http:// www.elm.chalmers.se/fie97/papers/1204.pdf

University of California - Santa Cruz. Science Library. (Page consultée le 25 février 1998). Science library classes \& workshops. [En ligne]. Adresse URL: http ://bob.ucsc.edu/library/science/scibi.html 